\title{
A Fully Implicit, Lower Bound, Multi-axial Solution Strategy for Direct Ratchet Boundary Evaluation: Theoretical Development
}

\author{
Alan Jappy \\ University of Strathclyde \\ Glasgow, United Kingdom \\ Email: alan.jappy@strath.ac.uk \\ Donald Mackenzie \\ University of Strathclyde \\ Glasgow, United Kingdom \\ Email: d.mackenzie@strath.ac.uk \\ Haofeng Chen \\ University of Strathclyde \\ Glasgow, United Kingdom \\ Email: haofeng.chen@strath.ac.uk
}

\section{ABSTRACT}

Ensuring sufficient safety against ratchet is a fundamental requirement in pressure vessel design. Determining the ratchet boundary can prove difficult and computationally expensive when using a full elastic-plastic finite element analysis and a number of direct methods have been proposed that overcome the difficulties associated with ratchet boundary evaluation. Here, a new approach based on fully implicit Finite Element methods, similar to conventional elastic-plastic methods, is presented. The method utilizes a two-stage procedure. The first stage determines the cyclic stress state, which can include a varying residual stress component, by repeatedly converging on the solution for the different loads by superposition of elastic stress solutions using a modified elastic-plastic solution. The second stage calculates the constant loads which can be added to the steady cycle whilst ensuring the equivalent stresses remain below a modified yield strength. During stage 2 the modified yield strength is updated throughout the analysis, thus satisfying Melan's Lower bound ratchet theorem. This is achieved utilizing the same elastic plastic model as the first stage, and a modified radial return method. The proposed methods are shown to provide better agreement with upper bound ratchet methods than other lower bound ratchet methods, however limitations in these are identified and discussed. 


\section{NOMENCLATURE}

a Used for calculating $\mathrm{X}$

b Used for calculating $\mathrm{X}$

c Used for calculating $\mathrm{X}$

$C_{i j k l} \quad$ Elastic constitutive matrix

E Elastic Modulus

$f \quad$ Yield function

$q_{\alpha} \quad$ internal hardening variables

$r_{i j} \quad$ Plastic strain normal directions

X Used to scale the constant stress for stage 2 ratchet analysis

$\delta \quad$ Partial derivative

$\delta_{i j} \quad$ Kroneker delta

$\rho_{i j}^{p} \quad$ Residual stress from constant load during stage 2

$\bar{\rho}_{i j} \quad$ Constant cyclic residual stress

$\rho_{i j}^{r} \quad$ Varying cyclic residual stress

$\widehat{\sigma}_{i j} \quad$ Elastic cyclic stress in equilibrium with the cyclic loads at a given point in the load cycle

$\hat{\sigma}_{i j}^{c} \quad$ Elastic constant stress in equilibrium with the constant load

$\sigma_{i j}^{p} \quad \rho_{i j}^{p}+\hat{\sigma}_{i j}^{c}$, Stress at loaded condition during stage 2

$\sigma^{\mathrm{y}} \quad$ Equivalent yield stress

$\sigma^{\mathrm{y}, \text { mod }}$ Equivalent yield stress used in stage 2 limit analysis

$\mu \quad$ Shear modulus

$\varepsilon_{i j}^{T} \quad$ Total mechanical strain

$\varepsilon_{i j}^{p} \quad$ Plastic strain

$\lambda \quad$ Lame's constant

$\gamma \quad$ Equivalent plastic strain

Indicies

$\mathrm{i}, \mathrm{j}, \mathrm{k}, \mathrm{l}$,

m,n,o, Are equal to 1,2 ,and 3

$\mathrm{p}$

$\alpha \quad$ Possible value are $1,2,3 \ldots$ to the number of internal hardening variables

Subscripts

1,2,3 Principle stress directions

Superscripts

Deviatoric component 


\section{INTRODUCTION}

Designing against ratcheting is a fundamental requirement in pressure vessel design. Bree [1] showed that the shakedown and ratchet boundaries of a simplified representation of an internally pressurized pipe subjected to a thermal cycle can be determine analytically. The Bree diagram summarizes the five possible structural responses to application of a constant and varying load, as shown in Fig. 1, these can be summarized as follows:

Fully Elastic: On the application of the constant and varying load no yielding is caused anywhere in the structure and all subsequent cycles are also fully elastic.

Elastic shakedown: On initial application of the constant and varying load some yielding occurs in one or more parts of the structure. However on cycling the varying load the structure achieves shakedown to a fully elastic strain cycle throughout the structure.

Plastic Shakedown: On the initial application of the constant and varying load, some yielding occurs in the structure. On cycling the load the structure will shakedown to a steady cycle which includes, at some location in the structure, a fully reverse plastic strain cycle.

Ratchet: On initial loading of the structure by the constant and varying load, yielding occurs in the structure. On cycling the varying load a closed cycle of strain is not achieved. This results in a net increase in the plastic strain during each subsequent cycle, leading to eventual incremental plastic collapse of the structure.

Limit: On the initial loading of the structure, the structure reaches its limit load on either the loading or unload part of the first cycle.

The long term response of the structure to a given load case can be simulated using incremental Finite Element Analysis but this approach does not directly define any of the boundaries, which must be established by trial and error. If the structural response is close to the ratchet boundary, the analysis may require a large number of cycles to demonstrate conclusive shakedown/ratchet behavior, making it difficult to judge whether or not shakedown will occur. A number of direct shakedown and ratchet methods, which make use of the finite element method and the theorems of Melan [2] and Koiter [3] have been proposed to overcome the difficulties associated with shakedown/ratchet boundary identification. In general, these methods are more successful in determining the boundary between the elastic shakedown and plastic shakedown zones, with the ratchet boundary proving more difficult to define. A number of approaches to determine the elastic shakedown boundary have been proposed, for example [4-8], however few of these methods have been extended to determine the plastic shakedown/ratchet boundary. In this paper, discussion is limited to direct ratchet methods that have been fully numerically implemented. Direct, in this context, refers to methods based on augmented limit analysis, although it is appreciated that other methods based on linear and non-linear programing methods are also available. Of the direct methods, those that have so far been shown to provide robust definition of the ratchet boundary have been the upper bound methods, most notably the Linear Matching Method (LMM) [9]. Here two new direct lower bound ratchet methods are proposed, these new methods aim to further understanding of the requirements for an accurate and reliable lower bound solution. 


\section{CONDITIONS TO PREVENT RATCHET}

Indicial notation is adopted, with the indices and their possible values given in the nomenclature section. Where indices are repeated, Einstein summation is implied. The term cyclic stress is used to refer to the stresses which result from the applied cyclic loads in the absence of any constant load.

A review of the conditions to satisfy strict shakedown and prevent the onset of ratchet is given by Pollizotto in [1013]. Here a summary of the necessary conditions to prevent ratchet are given.

Melan's lower bound [2] was originally developed as the necessary conditions for strict elastic shakedown of a structure subject to a load case involving a constant load and a cyclic load. However, Melan's lower bound theorem can be extended to the more general case of elastic and plastic shakedown. If a structure subject to constant load $\mathrm{P}^{\mathrm{c}}$ and time varying load $\widehat{P}(t)$, which can be described by fully elastic stresses $\widehat{\sigma}_{i j}^{c}$ and $\widehat{\sigma}_{i j}(t)$ respectively, these loads result in a constant, self-equilibrating, residual stress, $\rho^{p}+\bar{\rho}$, in addition to a time varying, self-equilibrating, residual stress $\rho^{\mathrm{r}}(\mathrm{t})$. Shakedown will occur if, under the combined action of the total residual stress $\rho^{p}+\bar{\rho}+\rho^{\mathrm{r}}(\mathrm{t})$ plus elastic stresses $\widehat{\sigma} \sigma_{\mathrm{ij}}^{\mathrm{c}}$ and $\widehat{\sigma}_{i j}(t)$, the yield conditions is not violated anywhere in the structure, with the time varying residual stress field vanishing over the cycle i.e.:

$\left|\rho^{p^{\prime}}+\bar{\rho}_{i j}^{\prime}+\rho_{i j}^{r^{\prime}}(t)+\hat{\sigma}_{i j}^{c^{\prime}}+\hat{\sigma}_{i j}^{\prime}(t)\right| \leq \sigma^{y}$

with

$\rho_{i j}^{r}(n \Delta t)=0 \quad[14]$

where $\mathrm{n}$ is an integer and $\Delta \mathrm{t}$ is the period of the varying load.

The time variant stress is added to allow for a reverse plasticity cycle. This stress is only allowed to develop in parts of the structure in which it vanishes to zero, i.e. it does not accumulate over the cycle. The problem then is how to identify parts of the structure which can support $\rho_{\mathrm{ij}}^{\mathrm{r} \prime}$ and then to evaluate $\rho_{\mathrm{ij}}^{\mathrm{r} \prime}(\mathrm{t})$ over the cycle.

The methods presented here are closely linked to the Hybrid method [15]. The Hybrid Method is based on the NonCyclic method [16] but uses direct cyclic analysis to determine the cyclic stresses which allow $\rho_{i j}^{r}(t)$ to vanish over the cycle, in a manner, similar to stage 1 of the Linear Matching Method [9], followed by an elastic plastic limit analysis with a yield strength modified based on the cyclic stresses. The basic solution strategy adopted in the methods proposed here is applied in two distinct stages. The first stage identifies the cyclic residual stress due to the cyclic stresses only, $\bar{\rho}_{\mathrm{ij}}+$ $\rho_{\mathrm{ij}}^{\mathrm{r}}(\mathrm{t})$, which allows the vanishing of the varying component, $\rho_{\mathrm{ij}}^{\mathrm{r}}(\mathrm{n} \Delta \mathrm{t})=0$. The second stage then uses the steady state cyclic stresses to perform an augmented limit analysis, similar to that used in the Hybrid method, in which the constant load at the ratchet boundary is equivalent to the constant load which causes limit collapse.

\section{UPPER AND LOWER BOUND RATCHET METHODS}

Direct ratchet boundaries methods fall into 2 groups: upper bound methods and lower bound methods. The Linear Matching Method (LMM) [9] and the Non-Cyclic method [16] are upper and lower bound methods respectively, that use 
an adjusted elastic modulus procedure similar to that of the elastic compensation method [17] to perform augmented limit analysis and hence identify the ratchet boundary. The reduced modulus procedure maintains incompressibility in the plastic strains but does not necessarily satisfy the associative plasticity assumption commonly used for metal plasticity. The methods differ in the process used to determine the cyclic stresses. The linear Matching Method utilizes the method in [18] whereby the cyclic stresses at each load point are matched back to yield and the resulting residual stresses are summed over the cycle to give the time invariant residual stress, whilst the non-cyclic method uses the method presented in [16] where a fully reversed cyclic load amplitude is applied to the model in an elastic plastic analysis to determine the redistributed cyclic stress amplitude which satisfies Melan's theorem. Consequently, the two methods use different cyclic stress descriptions in the limit analysis. Both methods have shown reasonable agreement on the ratchet boundary for a plate with hole subject to thermal cycle [16], however the LMM is more general in that it has been extended to multiextremes load cycles [18] and temperature dependent material properties through the use of a reference temperature [9]. A lower bound approximation of the LMM has also recently been proposed [19]. This uses the stresses generated by the upper bound LMM and scales them until they satisfy Melan's theorem. Despite using a well-developed upper bound solution, the lower bound approximation can be ambiguous and may require a relatively large number of iterations to converge.

The Hybrid method [15] and the simplified approaches used in the Uniform Modified Yield (UMY) and Load Dependent Yield Modification (LDYM) methods [20] are lower bound methods. The Hybrid method uses the Direct Cyclic Analysis shakedown method [21] currently incorporated in ABAQUS [22] to converge on the cyclic stresses with a vanishing $\rho_{i j}^{r}(t)$, and then utilizes a vector type summation of the stresses to calculate a yield stress and perform the augmented limit analysis. The Hybrid method calculates the yield stress at the beginning of the analysis only. If redistribution in the constant stress field occurs during the stage 2 limit analysis, the method could become overly conservative or non-conservative [15].

The simplified approaches used in the UMY and LDYM methods use a simplified description of the cyclic stress, proposed by Gokhfeld [15]. Whilst providing a relatively fast solution compared to other lower bound methods, the result presented in [20] show the LDYM can give lower bound solutions in excess of the calculated LMM upper bound solution. Therefore it is, at present, unclear whether this method provides a strict lower bound.

The methods proposed here differ from the Hybrid method in that elastic plastic finite element analysis is used throughout the analysis to converge on the residual stress. The yield strength for the limit analysis is updated on a per-iteration (proposed method 1) or per-increment (proposed method 2) basis, resulting in consistently lower bound estimates of the ratchet load. Details on the numerical algorithms used are presented in a companion paper [23].

It is noted that the Hybrid method is similar to the UMY method and the methods presented here are similar to the LDYM method, with the key difference being the treatment of the cyclic stresses. The addition of stage 1 to the Hybrid method and the methods presented here allows an estimate of the plastic strain range to be found, which is not available in the current UMY and LDYM methods.

\section{PROPOSED LOWER BOUND METHOD}


The proposed methods are based on 5 basic assumptions:

1:

The load history can be decomposed into constant and cyclic parts

2:

The cyclic loads lie within the ratchet boundary

3:

The constant load is applied under load control

4:

Perfect plasticity is assumed throughout

$5:$

Material properties are temperature independent.

If the load history can be decomposed into constant and cyclic components, the method of determining the ratchet boundary can be split into two parts. The first part is to determine the cyclic stress solution for the cyclic loads only, which can be found in the absence of the constant load [14], resulting in the vanishing of $\rho_{i j}^{r}(t)$. The second part is to determine the additional constant load the structure can support before the onset of ratchet. The method proposed here follows this structure, with "stage 1" used to determine the cyclic stress solution and "stage 2" to calculate the lower bound constant load magnitude for the onset of ratchet.

\subsection{STAGE 1 CONSITUTIVE MODEL}

Stage 1 of the proposed method is similar to the LMM and the Hybrid methods and is used to determine the cyclic stress state which results in vanishing $\rho_{i j}^{r}(t)$; that is, an estimate of the cyclic stress states which take place in the structure at the shakedown condition minus the stress from the constant load. The necessary but insufficient conditions the cyclic stresses have to satisfy in an upper bound solution are stated in Section 2. Using these conditions, a constitutively accurate elasto-plastic model is formulated, which allows convergence of the part of the constant residual stress state due to the cyclic loads. The same outcome can be achieved using a full elastic-plastic finite element analysis for the cyclic loads only, converging on the loaded state. However, in doing so the solution could require a large number of steps to achieve convergence, thus allowing the possibility of input errors. By converging on the residual stress state, the finite element model retains the appropriate boundary conditions but remains fully unloaded throughout stage 1 and stage 2 . The stresses from the cyclic loads are applied by superposition of fully elastic stresses determined from a separate analysis onto the yield function used for the calculation of the plastic strain increments. The direct cyclic procedure could also be used to determine the steady cyclic stresses, however reading the output database from the direct cyclic analysis is a bottleneck in the process that can be avoided using the method proposed below.

From assumptions 1 and 2, the cyclic stresses which result in vanishing $\rho_{i j}^{r}(t)$ may be found by repeated convergence on cyclic parts of the residual stress, $\bar{\rho}+\rho^{\mathrm{r}}(\mathrm{t})$, for each point in the cycle in turn. If assumption 2 is not valid, stage 1 will fail to converge on a closed cycle and a net increase in plastic strain will result. This could occur in circumstances in which the cyclic loads have primary stress contributions. This is also true for the Hybrid and LMM methods. 
The cyclic stresses are not limited to being fully elastic, as the proposed method allows reverse plasticity in parts of the structure, which when subject to the cyclic stresses, can sustain a reverse plasticity cycle whilst maintaining equilibrium. Converging on the residual stress, the constitutive equation becomes:

$\dot{\rho}_{l j}+\dot{\rho}_{l j}^{r}=C_{i j k l} \dot{\varepsilon}_{k l}^{e}=C_{i j k l}\left(\dot{\varepsilon}_{k l}^{T}-\dot{\varepsilon}_{k l}^{p}\right)$

where:

$C_{i j k l}=\lambda \delta_{i j} \delta_{k l}+\mu\left(\delta_{i k} \delta_{j l}+\delta_{i l} \delta_{j k}\right)$

in which $\lambda=\frac{v E}{(1+v)(1-2 v)}$ and $\mu=\frac{E}{2(1+v)}$

The plastic strains and stresses must satisfy the Kuhn-Tucker loading/unloading conditions:

$\dot{\gamma} \geq 0, \quad f \leq 0, \quad \dot{\gamma} f=0$

From the Kuhn-Tucker loading conditions on yielding $(\dot{\gamma}>0)$ the stresses must satisfy the yield condition:

$f=0$

The condition that on yielding $f=0$, also gives the consistency condition i.e. during yielding. $(\dot{\gamma}>0$ and $f=0)$ :

$\dot{f}=0$

The plastic strains are assumed to be such that:

$\dot{\varepsilon}_{k l}^{p}=\dot{\gamma} r_{i j}$

Assuming perfect plasticity the yield condition becomes, with superimposed elastic stresses:

$f=\left|\bar{\rho}_{i j}^{\prime}+\rho_{i j}^{r \prime}+\hat{\sigma}_{i j}^{\prime}\right|-\sigma^{y}=0$

where $\hat{\sigma}_{i j}$ is a fixed elastic stress, which is in equilibrium with the applied cyclic loads at a given point in the load cycle.

An expression for $\gamma$ may be found by expanding the consistency condition:

$\dot{f}=\frac{\delta f}{\delta \sigma_{i j}} \dot{\sigma}_{i j}+\frac{\delta f}{\delta q_{\alpha}} \dot{q}_{\alpha}$

where, assuming perfect plasticity, there are no internal hardening variables:

$\dot{f}=\frac{\delta f}{\delta \sigma_{i j}} \dot{\sigma}_{i j}$

As the model assumes rate independence, this may be expressed as:

$f=\frac{\delta f}{\delta \sigma_{i j}} C_{i j k l}\left(\varepsilon_{k l}^{T}-\varepsilon_{k l}^{p}\right)=\frac{\delta f}{\delta \sigma_{i j}} C_{i j k l}\left(\varepsilon_{k l}^{T}-\gamma r_{k l}\right)=0$

Rearranged gives: 
$\gamma=\frac{\frac{\delta f}{\delta \sigma_{i j}} C_{i j k l} \varepsilon_{k l}^{T}}{\frac{\delta f}{\delta \sigma_{m n}} C_{m n o p} r_{o p}}$

The above conditions can be interpreted as follow. For the von Mises yield condition, consider the stress state shown in Fig. 2. The residual stress state $\left(\bar{\rho}+\rho^{r}\right)$ at a point in the load cycle is described by point A. By superposition of a cyclic elastic stress $(\widehat{\sigma})$, which is in equilibrium with the applied loads at that point in the load cycle, the elastically loaded cyclic stress state $(\sigma)$ is then described by point B.

For the case shown in Fig. 2, in which the stress $\sigma \leq \sigma^{\mathrm{y}}$, the elastic solution provides a cyclic residual stress, $\bar{\rho}+\rho^{\mathrm{r}}$, which satisfies the lower bound ratchet conditions given in Section 2 equations 1 and 2, and the above constitutive conditions in equations 3-13. For the case where $\sigma>\sigma^{\mathrm{y}}$, the yield condition has been violated and a nonlinear elastoplastic model is used to return the stress to the yield surface, as illustrated in fig. 3.

The above conditions can then be recast in terms of von Mises yield condition with associative plastic flow. The stresses and strain are considered to be such that:

$\bar{\rho}_{i j}+\rho_{i j}^{r}=C_{i j k l}\left(\varepsilon_{k l}^{T}-\varepsilon_{k l}^{p}\right)$

The equivalent von Mises stress is assumed to describe the yielding behavior such that:

$\left|\sigma_{i j}^{\prime}\right|=\left|\bar{\rho}_{i j}^{\prime}+\rho_{i j}^{r^{\prime}}+\hat{\sigma}_{i j}^{\prime}\right|=\sqrt{\frac{3}{2}\left(\bar{\rho}_{i j}^{\prime}+\rho_{i j}^{r}+\hat{\sigma}_{i j}^{\prime}\right)\left(\bar{\rho}_{i j}^{\prime}+\rho_{i j}^{{ }^{\prime}}+\hat{\sigma}_{i j}^{\prime}\right)}$

The stress $\sigma$ at each point in the load cycle must satisfy the yield condition and the Kuhn-Tucker loading conditions given in equations 17 to 21 .

$f=\sqrt{\frac{3}{2} \sigma_{i j}^{\prime} \sigma_{i j}^{\prime}}-\sigma^{y}=\sqrt{\frac{3}{2}\left(\bar{\rho}_{i j}^{\prime}+\rho_{i j}^{r \prime}+\hat{\sigma}_{i j}^{\prime}\right)\left(\bar{\rho}_{i j}^{\prime}+\rho_{i j}^{r^{\prime}}+\hat{\sigma}_{i j}^{\prime}\right)}-\sigma^{y} \leq 0$

$\gamma \geq 0$

$\gamma f=0$

Assuming, associative plastic flow:

$\delta \varepsilon_{i j}^{p}=\delta \gamma r_{i j}$

$r_{i j}=\frac{\delta f}{\delta \sigma_{i j}}=\frac{3}{2} \frac{\bar{\rho}_{i j}^{\prime}+\rho_{i j}^{r^{\prime}}+\hat{\sigma}_{i j}^{\prime}}{\sqrt{\frac{3}{2}\left(\bar{\rho}_{i j}^{\prime}+\rho_{i j}^{r^{\prime}}+\hat{\sigma}_{i j}^{\prime}\right)\left(\bar{\rho}_{i j}^{\prime}+\rho_{i j}^{r^{\prime}}+\hat{\sigma}_{i j}^{\prime}\right)}}$

In addition to the above conditions perfect plasticity is assumed throughout such that $\sigma^{y}$ is constant, along with the consistency condition $\dot{f}=0$ giving the equivalent plastic strain increment as: 
$\gamma=\frac{r_{i j} C_{i j k l} \varepsilon_{k l}^{T}}{r_{m n} C_{m n o p} r_{o p}}=\frac{r_{i j} C_{i j k l} \varepsilon_{k l}^{T}}{3 \mu}$

The above conditions allow the development of stress update algorithms for $\bar{\rho}+\rho^{r}$ through an appropriate choice of integration scheme for $\gamma$. The integration scheme chosen here is the radial return method. The relevant stress update and integration schemes are given in [23]. The results obtained by stage 1 can also be used to obtain an estimate of the plastic strain range due to the cyclic load by simple consideration of the plastic strains which result at the converged state of stage 1.

When using the radial return method it is necessary to use a consistent tangent modulus to preserve the asymptotic rate of convergence of the global Newton-Raphson procedure. When defining the consistent tangent modulus for a particular iteration, i.e. for a particular point on the cycle it is necessary to use:

$\frac{\delta\left(\bar{\rho}_{i j}+\rho_{i j}^{r}\right)}{\delta \varepsilon_{k l}^{T}}=\frac{\delta \sigma_{i j}}{\delta \varepsilon_{k l}^{T}}-\frac{\delta \hat{\sigma}_{i j}}{\delta \varepsilon_{k l}^{T}}$

where for the given point on the load cycle, i.e. for a point in time:

$\frac{\delta \hat{\sigma}_{i j}}{\delta \varepsilon_{k l}^{T}}=0$

giving:

$\frac{\delta\left(\bar{\rho}_{i j}+\rho_{i j}^{r}\right)}{\delta \varepsilon_{k l}^{T}}=\frac{\delta \sigma_{i j}}{\delta \varepsilon_{k l}^{T}}$

This allows the rate of change of the point $\mathrm{A}$ to be described by the rate of change of point $\mathrm{B}$. This then allows use of a consistent tangent modulus in which the normal directions can be assumed to be constant during the increment, simplifying the derivation of the consistent tangent modulus.

By continually converging for multiple load cycles on $\bar{\rho}+\rho^{\mathrm{r}}$ for each point on the load cycle in turn, $\bar{\rho}$ will tend toward the cyclic solution with each $\rho^{\mathrm{r}}$ also tending toward the cyclic solution, which allows $\rho^{r}(t)$ to vanish over the cycle. When the difference between $\bar{\rho}+\rho^{\mathrm{r}}$ and $\varepsilon^{p}$ at every point in the load cyclic from one cycle to the next is small, the solution can be taken as converged.

The above model requires that a both limit state and stable cycle are possible with the given loads. In the case where the cyclic stress solutions are not within a limit state, the solution will fail to converge on the first load cycle.

\subsection{STAGE 2 CONSTITUTIVE MODEL}

Stage 2 of the proposed method calculates the magnitude of the "additional" constant load the structure can support without the onset of ratchet. Using the conditions for the constant load set out in Section 2, the constitutive model can be developed in a similar manner to stage 1 . In equations $25-39$, stage 2 is formulated using the loaded stress state to 
illustrate the calculation of the modified yield stress. To maintain the use of the stage 1 finite element model, stage 2 is then reformulated in terms of the residual stress in equations 40-48.

The strains used during stage 1 are reset back to zero everywhere in the structure before the start of stage 2 ; that is, the strains in stage 1 are not carried over to stage 2 . The constitutive model for the loaded point during the stage 2 ratchet analysis, using a new set of strains, is:

$\dot{\sigma}_{i j}^{p}=C_{i j k l} \dot{\varepsilon}_{k l}^{e}=C_{i j k l}\left(\dot{\varepsilon}_{k l}^{T}-\dot{\varepsilon}_{k l}^{p}\right)$

where:

$C_{i j k l}=\lambda \delta_{i j} \delta_{k l}+\mu\left(\delta_{i k} \delta_{j l}+\delta_{i l} \delta_{j k}\right)$

in which $\lambda=\frac{v E}{(1+v)(1-2 v)}$ and $\mu=\frac{E}{2(1+v)}$

The plastic strains and stresses must also satisfy the Kuhn-Tucker loading/unloading conditions. The plastic strains are assumed to be such that:

$\dot{\varepsilon}_{k l}^{p}=\dot{\gamma} r_{i j}$

Assuming perfect plasticity the yield condition becomes:

$f=\left|\bar{\rho}_{i j}{ }^{\prime}+\rho_{i j}^{r}{ }^{\prime}+\hat{\sigma}_{i j}{ }^{\prime}+\sigma_{i j}^{p^{\prime}}\right|-\sigma^{y}=0$

where $\sigma_{i j}^{p}$ is the stress in equilibrium with the constant load and $\bar{\rho}_{i j}{ }^{\prime}+\rho_{i j}^{r}{ }^{\prime}+\hat{\sigma}_{i j}{ }^{\prime}$ taken from stage 1 at each point in the load cycle. An expression for $\gamma$ may be found by expanding the consistency condition:

$\dot{f}=\frac{\delta f}{\delta \sigma_{i j}} \dot{\sigma}_{i j}+\frac{\delta f}{\delta q_{i j}} \dot{q}_{i j}$

where assuming perfect plasticity there are no internal hardening variables:

$\dot{f}=\frac{\delta f}{\delta \sigma_{i j}} \dot{\sigma}_{i j}$

As the model assumes rate independence:

$f=\frac{\delta f}{\delta \sigma_{i j}} C_{i j k l}\left(\varepsilon_{k l}^{T}-\varepsilon_{k l}^{p}\right)=\frac{\delta f}{\delta \sigma_{i j}} C_{i j k l}\left(\varepsilon_{k l}^{T}-\gamma r_{k l}\right)=0$

which rearranged gives:

$\gamma=\frac{\frac{\delta f}{\delta \sigma_{i j}} C_{i j k l} \varepsilon_{k l}^{T}}{\frac{\delta f}{\delta \sigma_{m n}} C_{m n o p} r_{o p}}$

In stage 2, the direction of plastic flow is assumed to be given by the constant load only. This is to ensure the direction of plastic flow is the same for each cyclic load case to allow the identification of a limiting case. If the cyclic stress is 
incorporated into the direction of plastic flow, as is suggested as further work in [23], the problem becomes more difficult as a limiting case, as all load cases cannot be given by direct comparison. In method 2, where the modified yield strength is calculated at the start of the increment, the integration scheme in [23] is associative, however for method 1, where the modified yield strength is calculated for every iteration, the integration scheme in [23] is non-associative, as:

$r_{i j}=\frac{\delta\left|\sigma_{i j}^{p^{\prime}}\right|}{\delta \sigma_{i j}^{p^{\prime}, \text { trial }}} \neq \frac{\delta f}{\delta \sigma_{i j}^{p^{\prime}, \text { trial }}}$

To be consistent with stage 1, the problem is formulated such that the model can be solved with the same radial return method used in stage 1 . This can be achieved through a supplementary calculation, similar to the Hybrid method [15], to determine the maximum equivalent constant stress the structure can support in addition to the cyclic stress for a given constant stress direction. This maximum equivalent constant stress is then used as the yield stress in a limit analysis with the constant loads only. This differs from the Hybrid method in that the maximum allowable constant equivalent stress is calculated during each iteration or at the start of each increment, to account for the redistribution in the constant stress field.

With the cyclic stresses calculated by stage 1 the constant stress must satisfy the following criteria, at every location in the structure at every point on the load cycle, to prevent the onset of ratchet:

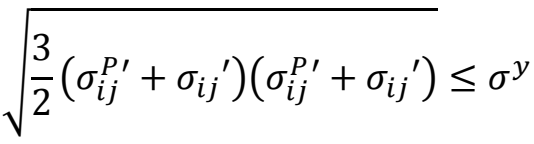

at every point in the load cycle.

For a given constant stress $\sigma_{i j}^{P}$ the maximum equivalent constant stress the structure can support without the onset of ratchet is given by:

$\sqrt{\frac{3}{2}\left(X{\sigma_{i j}}^{p \prime}+\sigma_{i j}{ }^{\prime}\right)\left(X{\sigma_{i j}}^{p \prime}+{\sigma_{i j}}^{\prime}\right)}=\sigma^{y}$

This gives the maximum equivalent stress from the constant load that the structure can support before the onset of ratchet as:

$\sigma^{y, \text { mod }}=\sqrt{\frac{3}{2}\left(X \sigma_{i j}^{p^{\prime}}\right)\left(X \sigma_{i j}^{p \prime}\right)}=X \sqrt{\frac{3}{2} \sigma_{i j}^{p^{\prime}} \sigma_{i j}^{p^{\prime}}}$

where $\mathrm{X}$ is given by:

$X=\frac{-b+\sqrt{b^{2}-4 a c}}{2 a}$

and: 
$a=\frac{3}{2} \sigma_{i j}^{p \prime} \sigma_{i j}^{p,} \quad b=3{\sigma_{i j}^{p}}^{p^{\prime}} \sigma_{i j}^{\prime} \quad$ and $c=\frac{3}{2} \sigma_{i j}{ }^{\prime} \sigma_{i j}{ }^{\prime}-\left(\sigma^{y}\right)^{2}$

This is illustrated pictorially in fig. 4 .

For a given trial constant stress, the above expression can be used to determine the maximum additional stress the structure can support for the given directions of stress. If $X \geq 1$, the structure can support a load greater than the currently applied load, thus the stress state satisfies the yield condition. If $\mathrm{X}<1$ then the structure cannot support the additional load without yielding and an elasto-plastic solution method must be used to return the equivalent constant load to $\sigma^{\mathrm{y}, \mathrm{mod}}$. This appears similar to the Hybrid method but here the extra load the structure is able to support is calculated at each iteration or increment, taking account of any redistribution in the constant stress state. Accounting for this redistribution produces more consistent results than the pure Hybrid method [23].

Using equation 37, the stress $\sigma^{p}$ is scaled down by reducing the deviatoric components. This is analogous to returning the stress to the yield surface during an elastic plastic analysis, as illustrated in Fig. 4. When there are numerous extremes in the load cycle, the direction of plastic strain caused by the constant load stays the same for each point in the load cycle and the plastic strain directions during stage 2 at every point in the load cycle:

$r_{i j}=\frac{\delta f}{\delta \sigma_{i j}^{p}}=\frac{3}{2} \frac{\sigma_{i j}^{p^{\prime}}}{\sqrt{\frac{3}{2} \sigma_{i j}^{p^{\prime}} \sigma_{i j}^{p^{\prime}}}}$

The value of $\mathrm{X}$ is calculated for each cyclic load plus constant load and the limiting case is taken, the limiting case being the smallest value of $\mathrm{X}$.

Whilst the above expression for $\mathrm{X}$ is sufficient to develop a model for stage 2 , it would require the finite element model to be loaded with the constant load, changing the Finite Element model part way through the analysis. Instead, the model will again converge on a residual stress, $\rho_{i j}^{p}$, which accounts for the redistribution in the constant stress field due to any plasticity in the structure; thus the same finite element model used for stage 1 can be used for stage 2 . As before, the residual stress field must be self-equilibrating.

The strains are not carried over from stage 1, thus stage 2 can be described by the following, converging on the residual stress:

$\rho_{i j}^{p}=C_{i j k l}\left(\varepsilon_{k l}^{T}-\varepsilon_{k l}^{p}\right)$

The equivalent von Mises stress is assumed to describe the yielding behavior such that, with superposition of the elastic stress in equilibrium with the applied constant loads:

$\left|\sigma_{i j}^{p \prime}\right|==\sqrt{\frac{3}{2}\left(\rho_{i j}^{p \prime}+\hat{\sigma}_{i j}^{c \prime}\right)\left(\rho_{i j}^{p \prime}+\hat{\sigma}_{i j}^{c \prime}\right)}$

The stress $\sigma_{i j}^{p}=\rho_{i j}^{p}+\hat{\sigma}_{i j}^{c}$ at each point in the load cycle must satisfy the yield condition and Kuhn-Tucker loading and unloading conditions, as given in equations 42-44. 
$f=\sqrt{\frac{3}{2} \sigma_{i j}^{p \prime} \sigma_{i j}^{p \prime}}-\sigma^{y, m o d}=\sqrt{\frac{3}{2}\left(\rho_{i j}^{p \prime}+\hat{\sigma}_{i j}^{c \prime}\right)\left(\rho_{i j}^{p \prime}+\hat{\sigma}_{i j}^{c \prime}\right)}-\sigma^{y, m o d} \leq 0$

$\gamma \geq 0$

$\gamma f=0$

Assuming, the plastic flow direction is given by the constant load:

$r_{i j}=\frac{\delta f}{\delta \sigma_{i j}^{p}}=\frac{3}{2} \frac{\sigma_{i j}^{p \prime}}{\sqrt{\frac{3}{2} \sigma_{i j}^{p \prime} \sigma_{i j}^{p \prime}}}=\frac{3}{2} \frac{\rho_{i j}^{p \prime}+\hat{\sigma}_{i j}^{c \prime}}{\sqrt{\frac{3}{2}\left(\rho_{i j}^{p \prime}+\hat{\sigma}_{i j}^{c \prime}\right)\left(\rho_{i j}^{p \prime}+\hat{\sigma}_{i j}^{c \prime}\right)}}$

gives:

$\gamma=\frac{r_{i j} C_{i j k l} \varepsilon_{k l}^{T}}{r_{m n} C_{m n o p} r_{o p}}=\frac{r_{i j} C_{i j k l} \varepsilon_{k l}^{T}}{3 \mu}$

Stage 2 can then be interpreted as shown in Fig. 5 and Fig. 6. In cases where $\left|\sigma_{i j}^{p \prime}\right|=\left|\rho_{i j}^{p \prime}+\hat{\sigma}_{i j}^{c \prime}\right| \leq \sigma^{y, \bmod }$, as shown in Fig. 5, the stresses satisfy all of the necessary conditions to prevent ratchet.

In cases where $\left|\rho_{i j}^{p}+\hat{\sigma}_{i j}^{c}\right|>\sigma^{y, m o d}$, an elastic plastic method is used to return $\sigma_{i j}^{p \prime}$ to the yield surface described by $\sigma^{y, \bmod }$ as shown in Fig. 6.

As with stage 1 if integrating the procedure with the radial return method describing the rate of change of stress $\rho_{i j}^{p}$ is complicated by the fact that the normal directions are not constant, it is easier to describe the rate of change of $\sigma_{i j}^{p}$ rather than $\rho_{\mathrm{ij}}^{p}$. By making use of:

$\frac{\delta \hat{\sigma}_{i j}^{c}}{\delta \varepsilon_{k l}^{T}}=0$

it can be shown:

$\frac{\delta \rho_{i j}^{p}}{\delta \varepsilon_{k l}^{T}}=\frac{\delta \sigma_{i j}^{p}}{\delta \varepsilon_{k l}^{T}}$

which allows the consistent tangent modulus to be defined by $\frac{\delta \sigma_{i j}^{p}}{\delta \varepsilon_{k l}^{T}}$ in which the normal directions stay constant during the iteration.

The above conditions describe a model that can be used to determine the ratchet boundary directly. The success of the model is dependent upon the integration procedure used to solve of the plastic strain increment. In the companion paper [23], the models will be integrated in a manner similar to the radial return method used in most Mises plasticity models.

Whilst this allows for the determination of the equivalent plastic strains, it is susceptible to the same stability problems as the radial return method. This is discussed in more detail in [23]. 


\section{APPLICATION TO PLATE WITH HOLE}

Using the algorithms developed in the companion paper [23], the constitutive theories defined above have been implemented in ABAQUS via the user subroutine UMAT. It is recognized that other Finite Element programs could also be used. Both method 1, which updates the yield stress each iteration, and method 2, which updates the yield stress at the start of each increment, are implemented in the same general solution procedure.

Step 1: Solve for elastic stress from constant load, write stress to state variable array

Step 2: Solve for elastic stress from first cycle extreme, write stress to state variable array $\vdots$

Step $1+n$ : Solve for elastic stress from $\mathrm{n}^{\text {th }}$ cycle extreme, write stress to state variable array

Step 2+n: Return model to unstressed state

Step 3+n: Perform stage 1, write cyclic stresses to state variable array

Step 4+n: Return model to unstressed state

Step 5+n: Perform stage 2

The method presented here is compared with the upper and lower bound LMM and the Hybrid method for a plate with a central hole subject to constant pressure and thermal cycle. The Hybrid method results were obtained by the authors using a variation of the proposed method and show good agreement with the results presented in [15]. The linear matching results were provided by James Ure, (see Acknowledgement).

The plate is square with edge length $\mathrm{L}$, as shown in Fig. 7. The hole is centrally located with a radius a, such that $a / L=0.1$. The depth $d$ of the plate is such that $d / L=0.005$. Symmetry is used to model only one eighth of the plate. The free ends of the plate are constrained to remain plane.

The load case analyzed is constant pressure $\mathrm{P}$ applied on the free ends plus temperature cycled throughout the structure from $\theta^{0}$ to $\theta(r, t)$, equation 49 , where $\theta^{0}$ is assumed to be ambient temperature.

$\theta(r, t)=\theta^{0}+\left(\Delta \theta(t)-\theta^{0}\right)^{\ln (5 a / r)} / \ln (5)$

The plate material properties are assumed to be temperature independent and isotropic. The elastic modulus $=$ 208GPa , Poisson's ratio $\mathrm{v}=0.3$, coefficient of thermal expansion $\alpha=5 \times 10^{-5}{ }^{\circ} \mathrm{C}^{-1}$ and yield strength $\sigma^{\mathrm{y}}=$ $360 \mathrm{MPa}$.

The ratchet boundaries obtained from several methods are shown in Fig. 8.

\section{DISCUSSION}

Figure 8 shows that the proposed method 1 becomes unstable at relatively low loads. A number of possible reasons for this are discussed in [23]. The proposed method 2, shows a more stable solution, similar to the lower bound linear 
Matching Method at a cyclic temperature ratio of 0.5. In general, method 2 finds a ratchet boundary more consistent with the upper bound LMM than the Hybrid method. This is attributed to the increase in modified yield strength in the majority of the structure that occurs on redistribution of the constant load, as illustrated in Fig. 9.

It can be seen that method 2, whilst counting for redistribution, starts to deviate significantly from the LMM results for temperature ratios above 0.5 but then tends toward the LMM results beyond a temperature ratio of approximately 1.5 . The cyclic stress solutions from stage 1 at a temperature ratio of 0.5 indicates that the region of residual stress from stage 1 is highly localized around the bore of the hole, with the residual stress elsewhere being relatively low as shown in Fig. 10.

The results at temperature ratio 1 show that regions of residual stress are starting to form at the edge of the plate, as shown in Fig. 11. When the cyclic temperature ratio is 2.5, the residual stress zones at the center and edges of the plate result in full reverse yielding at the residual state: i.e. cause reverse plasticity, as shown in Fig. 12. This suggests that when the residual stress is small, as in Fig. 10, the effect of the cyclic solution on the stage 2 ratchet analysis is negligible. As the cyclic temperature increases, the residual stress increases at the points where the constant load is applied. This appears to have a larger impact on stage 2. As the residual stress at the loaded faces becomes large and causes reverse plasticity, the effect this has on the ratchet analysis is reduced. This suggests that the regions of residual stress result in plastic strains that are not carried over to stage 2 . At a temperature ratio of 0.5 the region of plastic strain is localized at the bore of the hole and thus does not greatly affect the development of the constant load stress state. However, at a temperature ratio of 1 the plastic strains at the loaded faces could severely impact on the development of the constant load due to the plane-edge constraint of the Finite Element model. By removing the plastic strains from the cycle, the compatibility of the problem is not fully accounted for. Whilst compatibility is not a necessary required for Melan's theorem, it is enforced in the finite element analysis. When the temperature ratio is 2 , the regions of plasticity at the loaded faces result in reverse plasticity mechanisms and, in these methods, results in zero stiffness in this region and therefore the application of the constant load and will have a reduced impact on the compatibility of the problem. This suggests that not preserving the compatibility between stage 1 and stage 2 could be a source of the discrepancy between the proposed method and the LMM results. Further examples studied in [23] also suggest that plastic strain direction during stage 2 could also contribute to the difference between the methods. Further work is required to fully understand the effects of preserving compatibility and altering the description of plastic strain directions during stage 2.

\section{CONCLUSIONS}

A number of constitutive models which allow convergence on an estimate of the load conditions resulting in the onset of ratchet have been developed. From these models, stress update algorithms have been formulated in the companion paper [23] and a number of example problems investigated. These examples show that the per-iteration update of modified yield strength can result in instability during stage 2 and it is proposed to use the per-increment update of the 
yield strength given in method 2. Practical issues concerning instability and increment size are addressed in the companion paper [23].

The proposed method 2 has been shown to give a lower bound solution due to the use of a first stage to find the cyclic stresses and accounting for redistribution in the constant stress. However, whilst accounting for the redistribution in the stage 2 modified yield strength generally produces better agreement with the LMM results than the Hybrid method, the method cannot be said to be generally reliable. It is concluded that a limitation in the description of compatibility and plastic strain directions during the stage 2 ratchet analysis could be the reason for the differences between method 2 and the LMM. Further work is required to improve the description of compatibility and plastic strain directions during stage 2.

\section{ACKNOWLEDGEMENT}

The authors would like to acknowledge the contributions of Mr J Ure, University of Strathclyde, Glasgow for providing the lower and upper bound LMM results presented in Section 6.

\section{REFERENCES}

[1] Bree, J., 1967, "Elasto-plastic behaviour of thin tubes subjected to internal pressure and intermittent heat fluxes with application to fast reactor fuel elements," J. Strain. Analysis, 2, pp.226-238.

[2] Melan, E., 1936, “Theorie Statisch Unbestimmter Systeme aus Ideal-Plastischem Bastoff,". Sitzungsberichte der Akademie der Wissenschaft. Wien, Abtiia;145, pp.195-218.

[3] Koiter, W.T., 1960, “General theorems for elastic plastic solids.” Progress in solid mechanics, J.N. Sneddon and R. Hill, eds., North Holland, Amsterdam, 1, pp.167-221.

[4] Chen, H.F., 2010, "Lower and Upper Bound Shakedown Analysis of structures With Temperature-Dependent Yield Stress," J. of Pressure Vessel Technology, pp.132, 1-8

[5] Staat, M., Heitzer, M., 2001, "LISA a European Project for FEM-based Limit and Shakedown Analysis," Nuclear Engineering and Design, 206, pp.151-166.

[6] Muscat, M., Mackenzie, D. and Hamilton, R., 2003, "Evaluating shakedown by non-linear static analysis," Computers and Structures, 81, pp.1727-1737.

[8] Abdalla, H.F., Megahed, M.M., Younan, M.Y.A., 2007, “A Simplified Technique for Shakedown Limit Load Determination," Nuclear Engineering and Design, 237, pp.1231-1240.

[8] Mackenzie D, Boyle J T, Hamilton R., 2000, “The Elastic Compensation Method for Limit and Shakedown Analysis: a Review", The J. Strain Analysis for Engineering Design, 35 (3), pp.171-188.

[9] Chen, H.F., Ponter, A.R.S, 2010, “A Direct Method on the Evaluation of Ratchet Limit,” J. Pressure Vessel Technology, 132

[10] Pollizzotto, C., 1993, “On the Conditions to Prevent Plastic Shakedown of Structures: Part I- Theory,” Trans. ASME, J. Applied Mechanics, 60, pp.15-19. 
[11] Polizzotto, C., 1993, “On the Conditions to Prevent Plastic Shakedown of Structures: Part II- The Plastic Shakedown Limit Load,” Trans. ASME, J. Applied Mechanics, 60, pp.20-25.

[12] Pollizzotto, C., 1993, “A Study on Plastic Shakedown of Structures: Part I- Basic Properties,” Trans. ASME, J. Applied Mechanics, 60, pp.318-323

[13] Pollizzotto, C., 1993, “A Study on Plastic Shakedown of Structures: Part II- Theorems,” Trans. ASME, J. Applied Mechanics, 60, pp.324-330

[14] Gokhfeld, D.A. and Cherniavsky, O.F., 1980, Limit Analysis of Structures at Thermal Cycling, Sijthoff \& Noordhoff

[15] Martin, M., 2009, “A hybrid procedure for ratchet boundary prediction,” PVP2009-77474, Pressure Vessels and Piping, Prague, Czech Republic

[16] Adibi-Asi, R., Reinhardt, W., 2010, “Ratchet Boundary Determination Using a Noncyclic Method,” Trans. ASME, J. Pressure Vessel Technology; 132 (2)

[17] Mackenzie, D., Boyle, J. T., Hamilton, R., 2001, "The elastic compensation method for limit and shakedown analysis: A review,” The J. Strain Analysis for Engineering Design, 35 (3), pp.171-188.

[18] Chen, H., Ponter, A., 2001, "A method for the evaluation of a ratchet limit and the amplitude of plastic strain for bodies subjected to cyclic loading,” Eur. J. Mech. A/Solilds, 20, pp.555-571.

[19]Ure, J., Chen, H., Li T., Chen, W., Tipping, D., Mackenzie D., 2011, “ A Direct Method for the Evaluation of Lower and Upper Bound Ratchet Limits," International Conference on the Mechanical Behaviour of Materials. Lake Como, Italy

[20] Abou-Hanna, J., McGreevy, T. E., 2011, “A simplified ratchet limit analysis using modified yield surface,” Int. J. of Pressure Vessel and Piping, 88, 11-18

[21] Nguyen-Tajan, et al., 2003, "Determination of the stabilized response of a structure undergoing cyclic thermalmechanical loads by a direct cyclic method," Abaqus Users' Conference Proceedings.

[22] Abaqus 6.10, 2010, SIMULIA

[23] Jappy, A., Mackenzie, D., Chen, H., 2012, “A Fully Implicit, Lower Bound, Multi-axial Solution Strategy for Direct Ratchet Boundary Evaluation: Numerical Development,” PVP2012-78315, Toronto, Ontario, CANADA

\section{List of Figures}

FIG. 1: BREE DIAGRAM WITH INSET STRESS-STRAIN RESPONCES

FIG. 2: ELASTIC RESIDUAL AND LOADED STRESS VECTORS BELOW YIELD

FIG. 3: ELASTIC RESIDUAL AND LOADED STRESS VECTORS ABOVE YIELD

FIG. 4: CALCULATION OF THE MAXIMUM ALLOWABLE CONSTANT EQUIVALENT STRESS

FIG. 5: PICTORIAL REPRESENTATION OF STAGE $2, X \leq 1$

FIG. 6: PICTORIAL REPRESENTATION OF STGAE 2, $X>1$ 
FIG. 7: PLATE WITH HOLE

FIG. 8: RATCHET BOUNDARY PLATE WITH HOLE

FIG. 9: MODIFIED YIELD STRENGTH $\frac{\Delta \theta}{100}=0.5$ : LEFT METHOD 2; RIGHT HYBRID METHOD

FIG.10: RESIDUAL STRESS, $|\bar{\rho}|$, AT A CYCLIC TEMPERATURE $\frac{\Delta \theta}{100}=0.5$

FIG. 11: RESIDUAL STRESS, $|\bar{\rho}|$, AT A CYCLIC TEMPERATURE $\frac{\Delta \theta}{100}=1.0$

FIG. 12: RESIDUAL STRESS, $|\bar{\rho}|$, AT A CYCLIC TEMPERATURE $\frac{\Delta \theta}{100}=2.5$ 


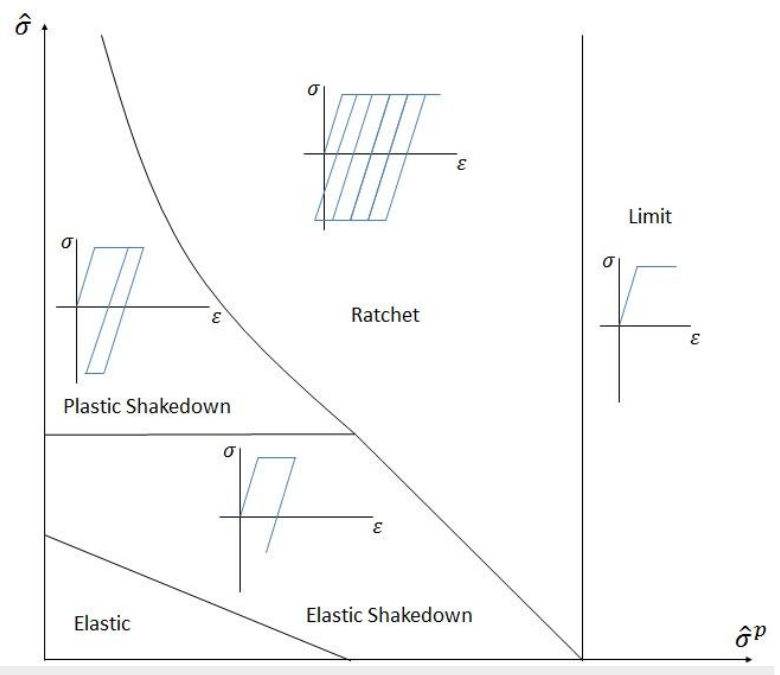

FIG. 1: BREE DIAGRAM WITH INSET STRESS-STRAIN RESPONCES 


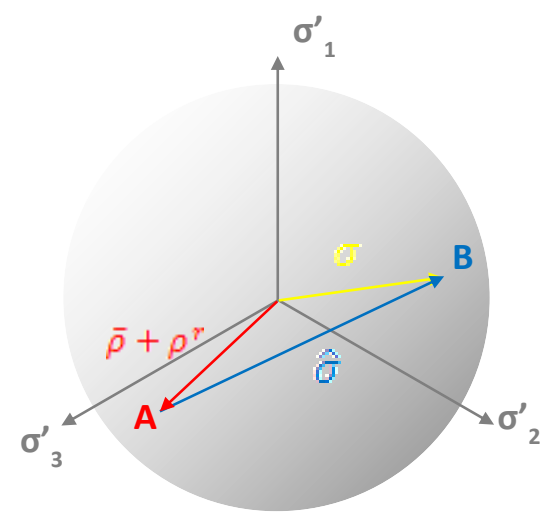

FIG. 2: ELASTIC RESIDUAL AND LOADED STRESS VECTORS BELOW YIELD 


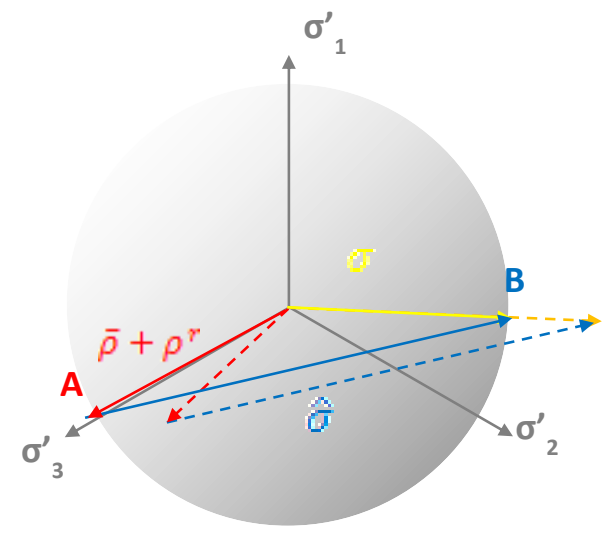

FIG. 3: ELASTIC RESIDUAL AND LOADED STRESS VECTORS ABOVE YIELD 


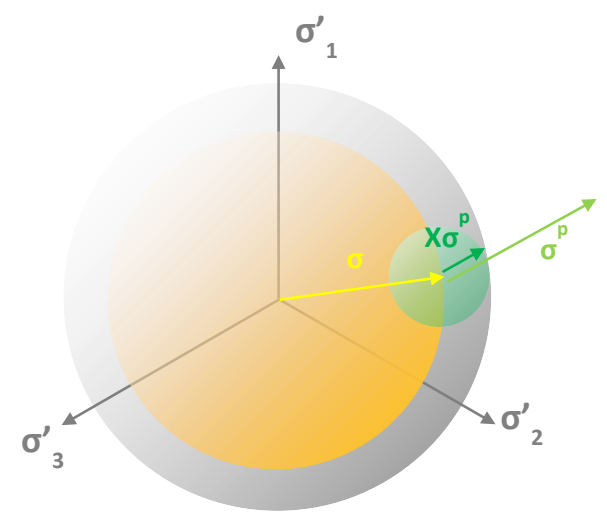

FIG. 4: CALCULATION OF THE MAXIMUM ALLOWABLE CONSTANT EQUIVALENT STRESS 


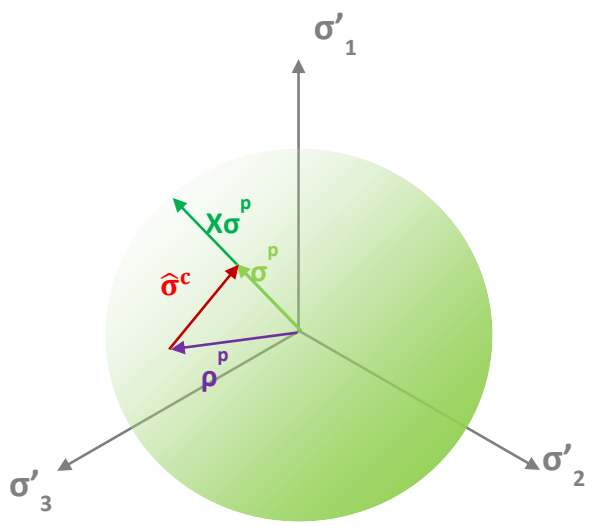

FIG. 5: PICTORIAL REPRESENTATION OF STAGE $2, X \leq 1$ 


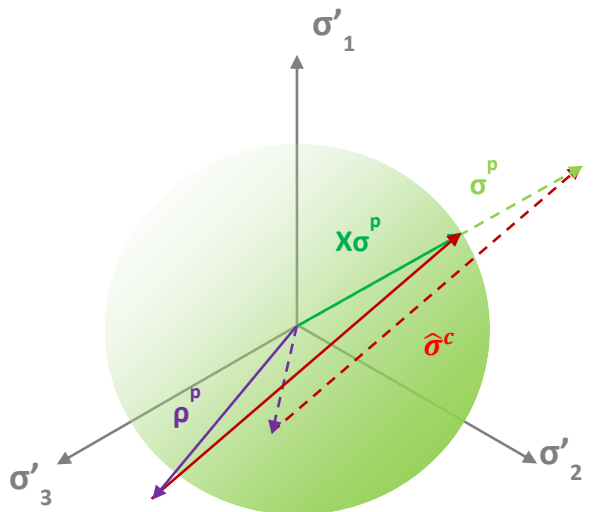

FIG. 6: PICTORIAL REPRESENTATION OF STAGE $2, X>1$ 


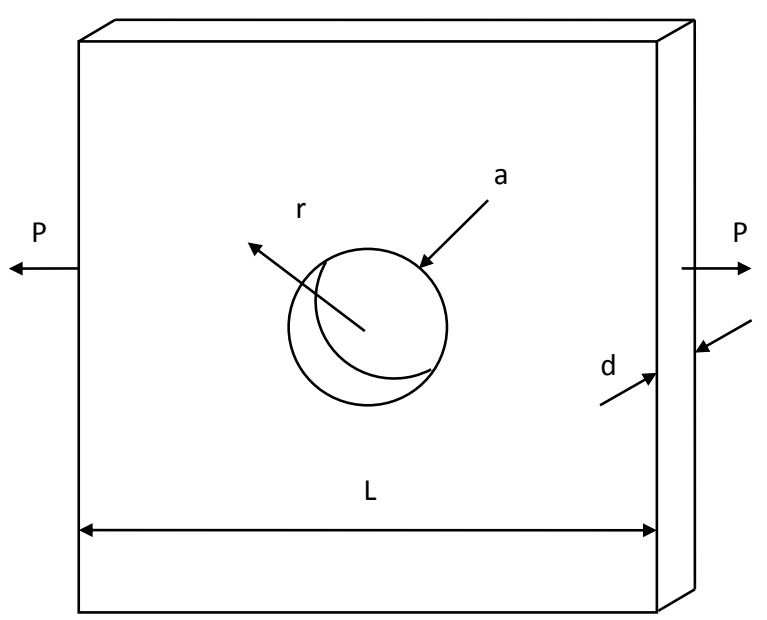

FIG. 7: PLATE WITH HOLE 


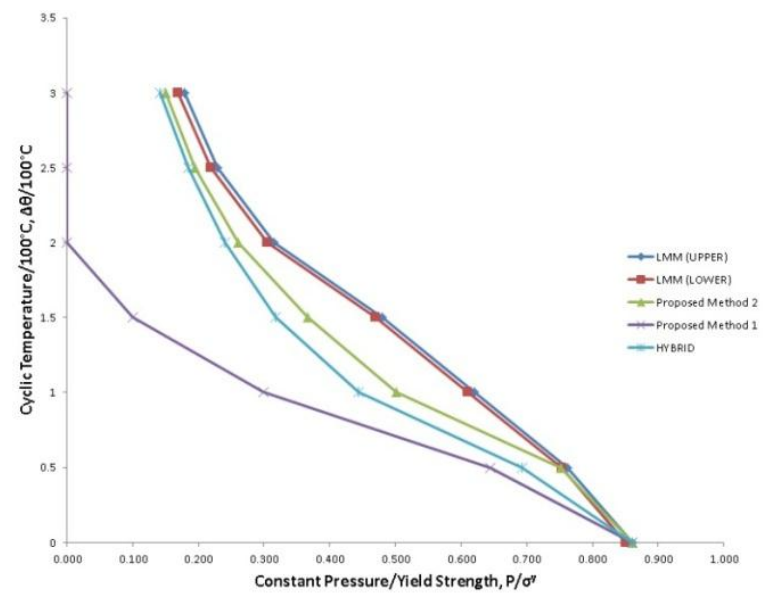

FIG. 8: RATCHET BOUNDARY PLATE WITH HOLE 


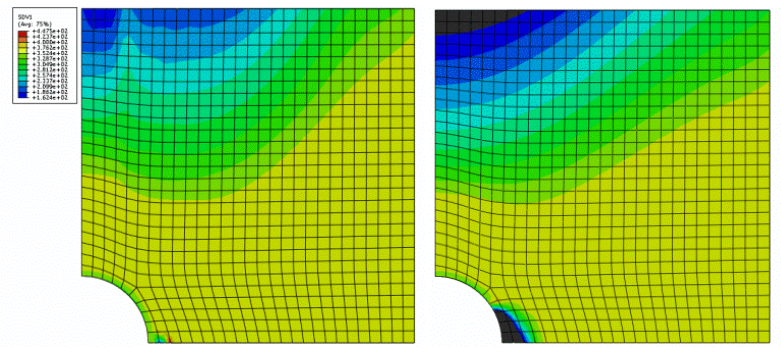

FIG. 9: MODIFIED YIELD STRENGTH $\frac{\Delta \theta}{100}=0.5$ : LEFT METHOD 2: RIGHT HYBRID METHOD 


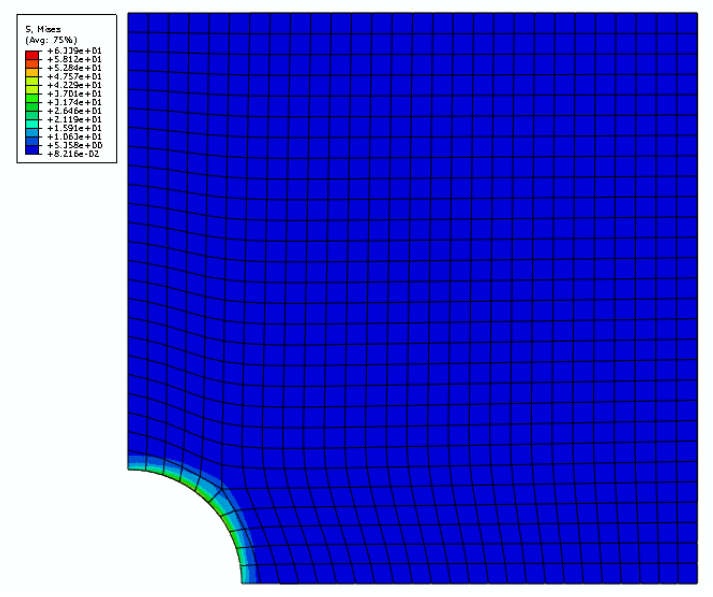

FIG. 10: RESIDUAL STRESS, $|\bar{\rho}|$, AT A CYCLIC TEMPERATURE $\frac{\Delta \theta}{100}=0.5$ 


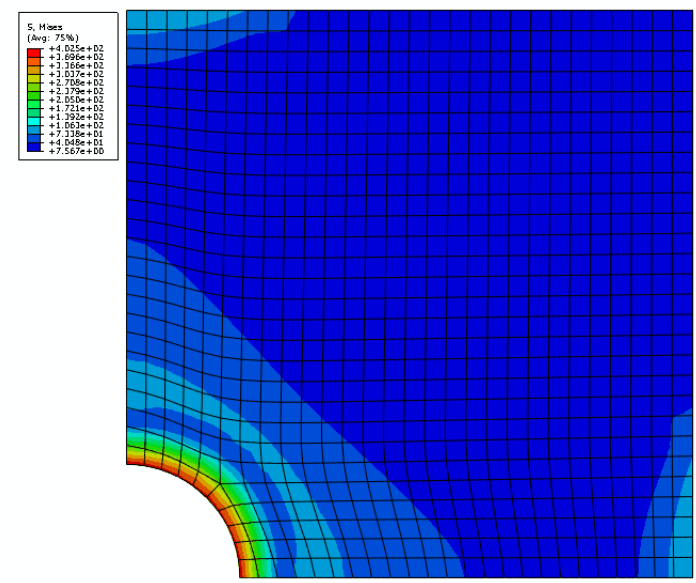

FIG. 11: RESIDUAL STRESS, $|\bar{\rho}|$, AT A CYCLIC TEMPERATURE $\frac{\Delta \theta}{\mathbf{1 0 0}}=\mathbf{1 . 0}$ 


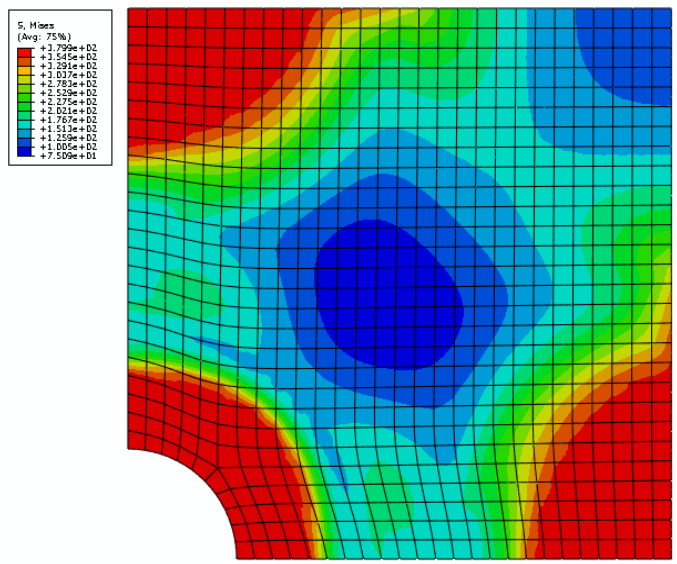

FIG. 12: RESIDUAL STRESS, $|\bar{\rho}|$, AT A CYCLIC TEMPERATURE $\frac{\Delta \theta}{100}=2.5$ 